\title{
The Use of Quantitative Image Analysis to Evaluate the Effectiveness of Microwave-Assisted Formaldehyde Fixation
}

\author{
R.T. Giberson
}

Research and Development, Ted Pella, Inc., 4595 Mountain Lakes Blvd., Redding, CA 96003

This research was made possible through the involvement of the Center for Comparative Medicine (CCM) and Department of Pathology and Laboratory Medicine, University of California Davis, Davis, CA. The Davis center is part of the National Cancer Institute's Mouse Models of Human Cancers Consortium and serves as the Center for Translational Genomic Phenotyping.

The purpose of this study was to test the efficacy of microwave-assisted formaldehyde fixation. Our previous studies demonstrated that variable microwave wattage and temperature control reduced the 24 hour bench fixation time with 10\% neutral buffered formalin (NBF) to under 1 hour without compromising morphology or vimentin antigenicity [1,2]. Tissues were fixed either in the microwave (PELCO BioWave ${ }^{\circledR}$ Pro, Ted Pella, Inc., Redding, CA) or passively on the bench for 21 hours. After fixation tissues were held in $70 \%$ ethanol prior to processing into paraffin with either a VIPTM or Tissue-Tek ${ }^{\circledR}$ automatic processor (Sakura Finetek USA, Inc., Torrance, CA). Tissues came from three mouse models of human breast cancers. Microwave-assisted NBF fixation was evaluated using the IHC4 antibody panel of estrogen receptor (ER), progesterone receptor (PR), Her-2/neu, and Ki-67 [3]. The three transplantable mouse breast tumor models used were: Met-1, a basal-like breast cancer negative for ER, PR, and Her-2/neu; SSM-2, a luminal-like breast cancer positive for ER, PR; and NDL line, a luminal-like breast cancer positive for ER, PR, and Her-2/neu.

Tissues fixed for 21 hours at room temperature in NBF were compared to tissues in NBF processed in the microwave at either $150 \mathrm{~W}$ for 60 minutes or at $150 \mathrm{~W}$ for 45 minutes followed by $650 \mathrm{~W}$ for 15 minutes. Temperature was kept constant in the microwave at $38^{\circ} \mathrm{C}$. Formalin fixed paraffin embedded sections were stained with the IHC4 panel used for the prognosis of human breast cancers [3]. Digitized whole slide image analyses was performed with the Aperio ScanScope XT in the Spectrum database using algorithms measuring nuclear staining intensities or by measuring membrane staining intensities per area by pixels. The number of negative and positive staining cells was calculated and staining intensity reported as,+++ or +++ . In this way an objective analysis of each processing method could be quantified and compared.

Results for microwave and 21-hour NBF fixation are shown for Ki-67 (Figure 1), a nuclear protein associated with cellular proliferation and ribosomal RNA transcription. Tissues were from the SSM2 mouse models. Figure 2 compares the total percent positive labeling for estrogen receptor in SSM-2 mice. Initial results from this study have indicated that microwave-assisted NBF fixation, as measured by IHC4 antibody panel for antigenicity, is comparable to traditional bench methods. The results of a single wattage $(150 \mathrm{~W})$ protocol (Figure 2 ) versus a two wattage strategy (Figure 1) are currently being evaluated. Other antigens are being examined beyond the IHC4 group as well.

Microwave processing methods have improved greatly over the last 40 years. The technology to change the continuous power output of the magnetron (variable wattage) and control sample temperature without turning off the magnetron have led to advancements in tissue fixation. The 
single or dual wattage approach to a microwave-assisted NBF fixation demonstrates that protocols can be customized for individual antigens.

References

[1] R.T. Giberson and D.E. Elliott, In: Microwave Techniques and Protocols, Humana Press, Inc., Totowa, NJ, 2001.

[2] J.J. Galvez et al., The J. Histotechnol. 29 (2006) 113.

[3] J. Cuzick et al., San Antonio Breast Cancer Symposium (Suppl. 74) (2009)

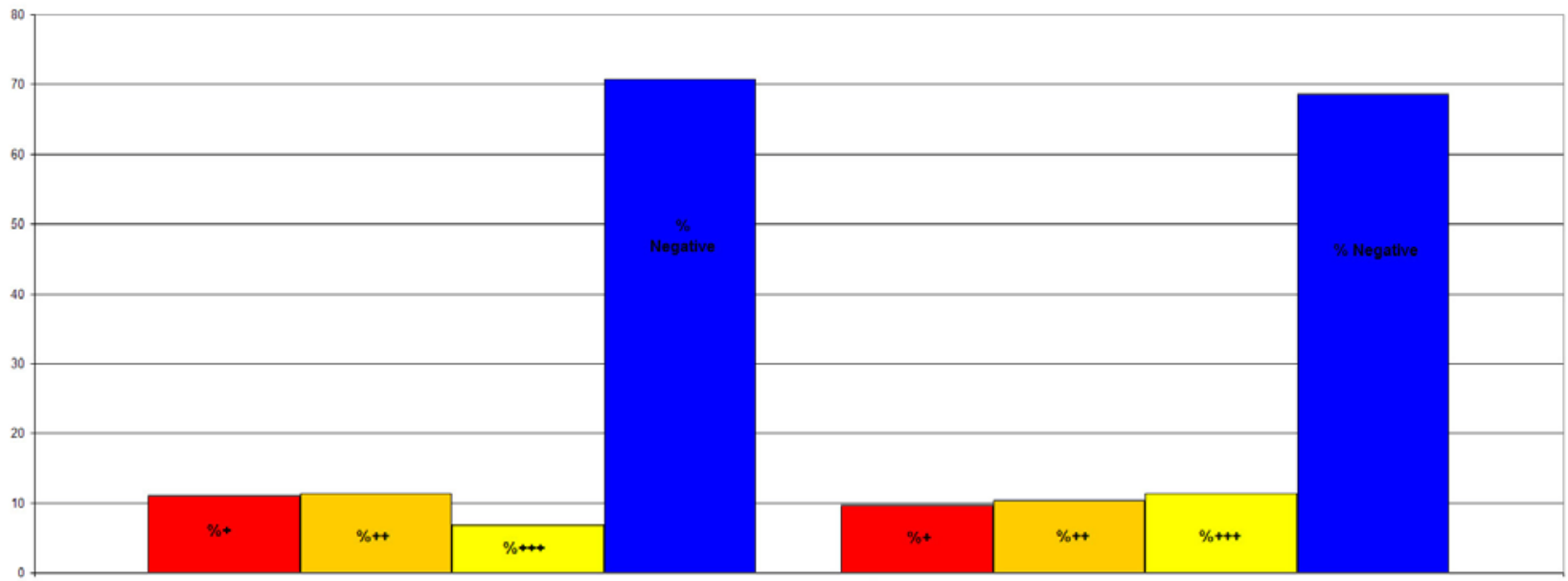

FIG. 1. The histogram on the left is the 21-hour control labeling intensities (red, gold, yellow) for $\mathrm{Ki}-67$ from the tumors of SSM-2 mice. The histogram on the right is from microwave $(150 \mathrm{~W} / 45 \mathrm{~min} .+650 \mathrm{~W} / 15 \mathrm{~min}$.) processed tissue from the same animal. Labeling intensities were determined from Aperio ScanScope XT digitized whole slide images of the tumors.

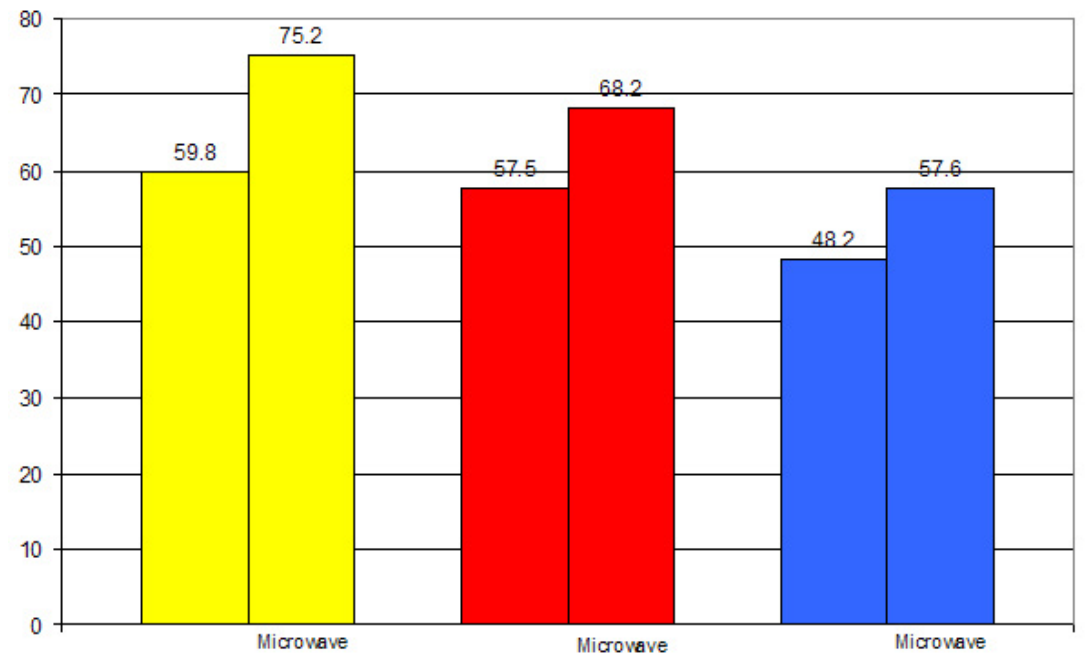

FIG. 2. This figure compares organ staining between $150 \mathrm{~W}$ microwave-assisted NBF fixation and the 21-hour control for whole organ positive staining for estrogen receptor. The yellow histograms represent staining in the uterus, the red histograms the mammary gland and the blue histograms the tumor. Labeling intensities were determined from Aperio ScanScope XT digitized whole slide images of the appropriate tissues. 